
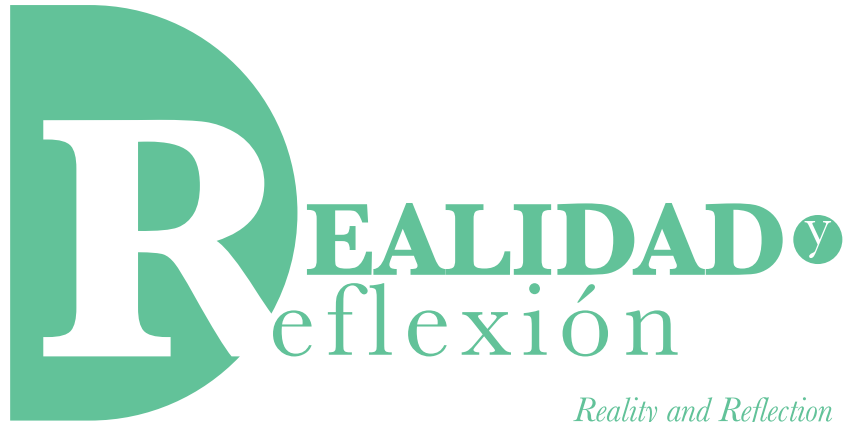

ISSN 1992-6510

e-ISSN 2520-9299

Reality and Reflection

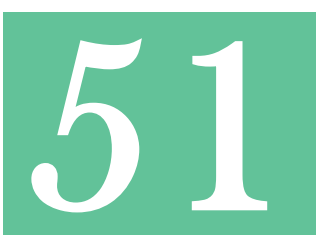

Año 20, No 51, San Salvador, El Salvador, Centroamérica. Revista Semestral Enero-Junio 2020

YEAR 20, N51, SAN SALVADOR, EL SALVADOR, CENTRAL AMERICA. SEMESTRAL JOURNAL JANUARY-JUNE 2020

\title{
Torneos parabólicos, torneos localmente transitivos y la curvatura de Ricci para torneos parabólicos
}

Parabolic tournaments, locally transitive tournaments and the Ricci curvature for parabolic tournaments

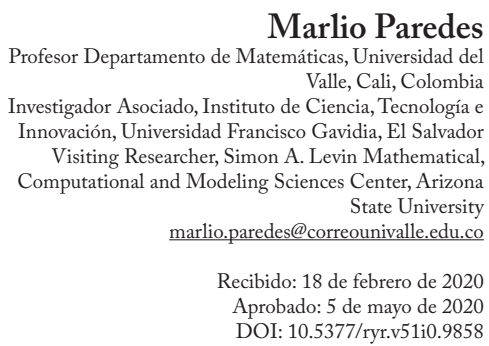

(c) (1) (8)(2) 


\section{RESUMEN}

Los torneos son un tipo de grafos dirigidos los cuales han sido usados para estudiar la geometría de variedades bandera clásicas, para lo cual se usa la relación existente entre torneos y estructuras cuasicomplejas sobre las variedades bandera clásicas. Elinterés porestudiar este tipo de grafos radica en la posibilidad de usar las propiedades combinatorias de los torneos para estudiar propiedades geométricas de las variedades bandera. En este artículo presentamos las ideas básicas sobre la curvatura de Ricci para torneos y, en particular, estudiamos algunas propiedades de los torneos parabólicos y los torneos localmente transitivos.

Palabras clave: Grafos dirigidos, torneos, curvatura de Ricci, torneos parabólicos, torneos localmente transitivos.

\section{ABSTRACT}

Tournaments are a type of directed graphs which have been used to study the geometry of classical fag manifolds, for which the existing relationship between tournaments and almost complex structures on classical flag manifolds is used. The interest to study this type of graphs liesin the possibility of using the combinatorial properties of tournamentsto study geometric properties of the flag manifolds. In this paper we present the basic ideas about the Ricci curvature for tournaments and in particular we study some properties of parabolic tournaments and locally transitive tournaments.

Keywords: Directed graphs, tournaments, Ricci curvature, parabolic tournaments, locally transitive tournaments. 


\section{Introducción}

Los torneos son una clase de grafos dirigidos para los cuales cualquier par de vértices está unido por exactamente un arco orientado.Este tipo de grafoshan sido usados para estudiar diferentes estructuras geométricas.Los primeros en usar torneos para estudiar la geometría de variedades bandera fueron Burstall y Salamon [2], ellos descubrieron la relación que existe entre estructuras cuasicomplejas sobre las variedades bandera clásicas y torneos.Con dicharelación ellos clasificaron todas las estructuras cuasicomplejas que admiten métricas Kähler sobre variedades bandera clásicas.

Posteriormente, Mo y Negreiros [17] usaron torneos para estudiar estructuras (1,2)-simplécticas sobre variedades bandera clásicas y con estas producir nuevos ejemplos de aplicaciones armónicas. A partir de ese trabajo surgieron varios trabajos en los cuales se usaron torneos entre los que podemos citar los de Cohen, Negreiros y San Martin [4,5], Cohen, Paredes y Pinzón [6], Florez [9], Paredes [22-25], Paredes, González y McKay [26], Paredes y Pinzón [27-30].

En 2011, Lin, Lu y Yau [14] modificaron la definición de curvatura de Ricci de cadenas de Markov dada por Olivier [21] e introdujeron el concepto de curvatura de Ricci para grafos. Ellos estudiaron propiedades de la curvatura de Ricci de grafos en general, del producto cartesiano de grafos y de grafos estocásticos. Más tarde, en [15], los mismos Lin, Lu y Yau estudiaron grafos Ricci-planos los cuales son aquellos para los que la curvatura de Ricci se anula en todos sus vértices. En [16], Lin y Yau dan algunas estimativas para la curvatura de Ricci y también obtienen una estimativa para el autovalor del operador de Laplace sobre grafos finitos.

Recientemente, Yamada [31] estudió la curvatura de Ricci de un grafo dirigido, en dicho trabajo se estudian algunas propiedades de la curvatura de Ricci y se encuentran condiciones para que un grafo dirigido regular sea plano. Además, se calcula la curvatura de Ricci del producto cartesiano de grafos dirigidos.

Usando ideas de Yamada [31], en este trabajo estudiamos la curvatura de Ricci para torneos. En particular analizamos el caso de los torneos parabólicos los cuales han sido usados para estudiar estructuras geométricas sobre las variedades bandera clásicas por Paredes y Pinzón [29]. Asimismo, presentamos un nuevo resultado que nos dice que todo torneo parabólico es localmente transitivo.

\section{Torneos parabólicos y torneos localmente transitivos}

Definición 2.1. Un torneo o $n$-torneo es un grafo dirigido o dígrafo con $n$ vértices o jugadores $p_{1}, \ldots, p_{n}$, tales que cada par de vértices está unido por exactamente un arco $p_{i} \rightarrow p_{j} o p_{j} \rightarrow p_{i}$. Si $p_{i} \rightarrow p_{j}$ decimos que $p_{i}$ le gana a $p_{j}$, ver [20]. 
Se acostumbra también definir un torneo como un par $T=(V, E)$, donde $V$ es el conjunto de vértices y $E$ el conjunto de arcos, con la condición que entre cada par de vértices siempre existe un único arco que los une. Asimismo, se acostumbra denotar los arcos como un $\operatorname{par}\left(p_{i}, p_{j}\right)$, si $p_{i} \rightarrow p_{j}$.

Definición 2.2. Dados los torneos $T_{1}$ con $n$ jugadores $\{1, \ldots, n\}$ y $T_{2}$ con $m$ jugadores $\{1, \ldots, m\}$, un homomorfismo entre $T_{1}$ y $T_{2}$ es una aplicación $\phi:\{1, \ldots, n\} \rightarrow\{1, \ldots, m\}$ tal que si $s \rightarrow t$ en $T_{1}$ entonces $\phi(s) \rightarrow \phi(t)$ ó $\phi(s)=\phi(t)$ en $T_{2}$. Cuando $\phi$ es biyectiva se dice que $T_{1}$ y $T_{2}$ son isomorfos.

Cada torneo determina una $n$-upla $\left(s_{1}, \ldots, s_{n}\right)$ cuyas entradas son el número de juegos que gana cada jugador. Esta $n$-upla es llamada vector resultado o vector marcador del torneo (en inglés score vector). Podemos ordenar las entradas del vector resultado de tal forma que $0 \leq s_{1} \leq \cdots \leq S n \leq n$ y además tenemos que $\sum_{i=1}^{n} s_{i}=\left(\begin{array}{l}n \\ 2\end{array}\right)$.

Los torneos pueden ser clasificados en clases de isomorfismo, la Figura 1 contiene las clases de isomorfismo de $n$-torneos para $n=2,3,4$. En esta figura se está usando la siguiente convención introducida por Moon [20], si se observa la figura vemos que faltan algunos arcos entre los vértices, pero en la convención de Moon se sobreentiende que desde cada vértice superior hay un arco yendo hacia cada vértice inferior.

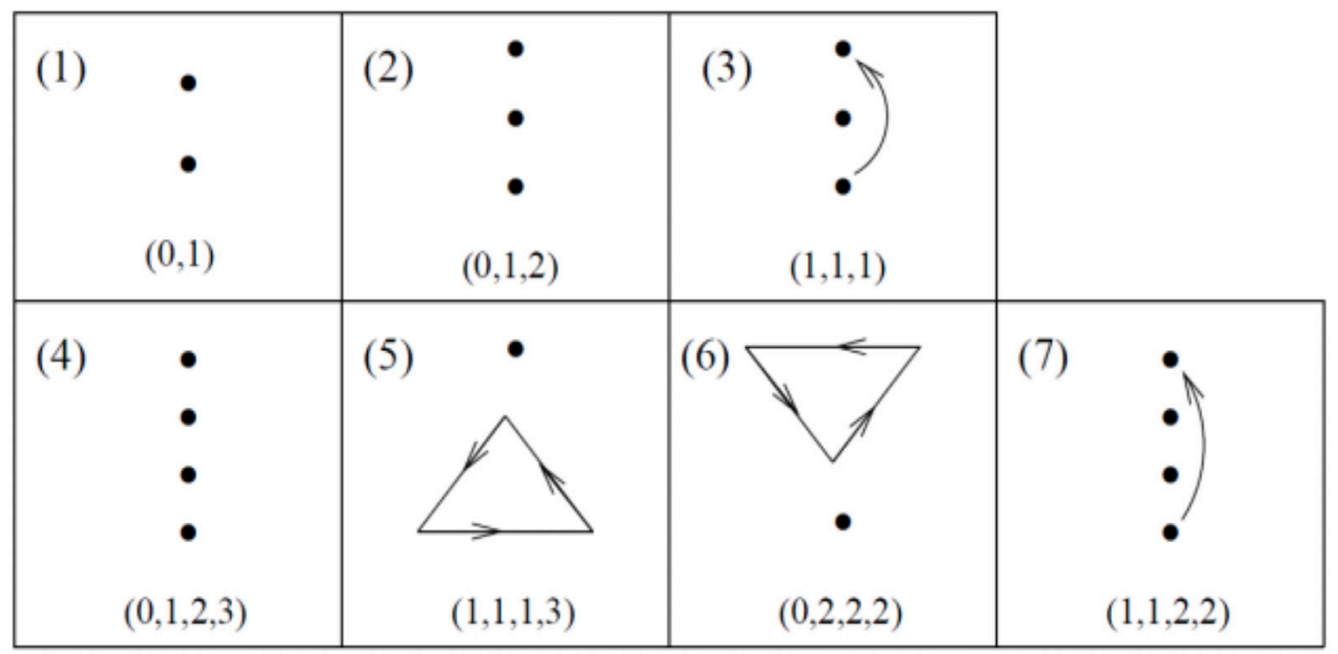

Figura 1. Clases de isomorfismo de $n$-torneos para $n=2,3,4$, y vector resultado correspondiente.

Claramente torneos isomorfos tienen el mismo vector resultado, pero no es cierto que torneos con el mismo vector resultado sean isomorfos como sucede con los 5-torneos (9), (10) y (11) en la Figura 2, los cuales tienen el mismo vector resultado $(1,2,2,2,3)$ pero no son isomorfos. 
Se dice que un torneo es transitivo si la relación “ $\rightarrow$ ” es transitiva, esto es, si $i \rightarrow j$ y $j \rightarrow k$ entonces $i \rightarrow k$. Los torneos transitivos tienen la particularidad que todos ellos son isomorfos entre si y su vector resultado es $(0,1,2, \ldots, n-1)$. Las clases de isomorfismo correspondiente para $n=2,3,4$ son respectivamente (1), (2) y (4) en la Figura 1 y para $n=5$ es (1) en la Figura 2.

El $n$-torneo canónico $T_{n}$ (ver [2]) es definido por

$\rightarrow j \quad \Leftrightarrow \quad i<j$

es fácil ver que salvo isomorfismos este torneo satisface las siguientes propiedades equivalentes

- $T_{n}$ es transitivo.

- $\quad T_{n}$ no contiene 3-ciclos, i.e. caminos cerrados $i_{1} \rightarrow i_{2} \rightarrow i_{3} \rightarrow i_{1}$.

- $\quad$ El vector resultado de $T_{n}$ es $(0,1,2, \ldots, n-1)$.

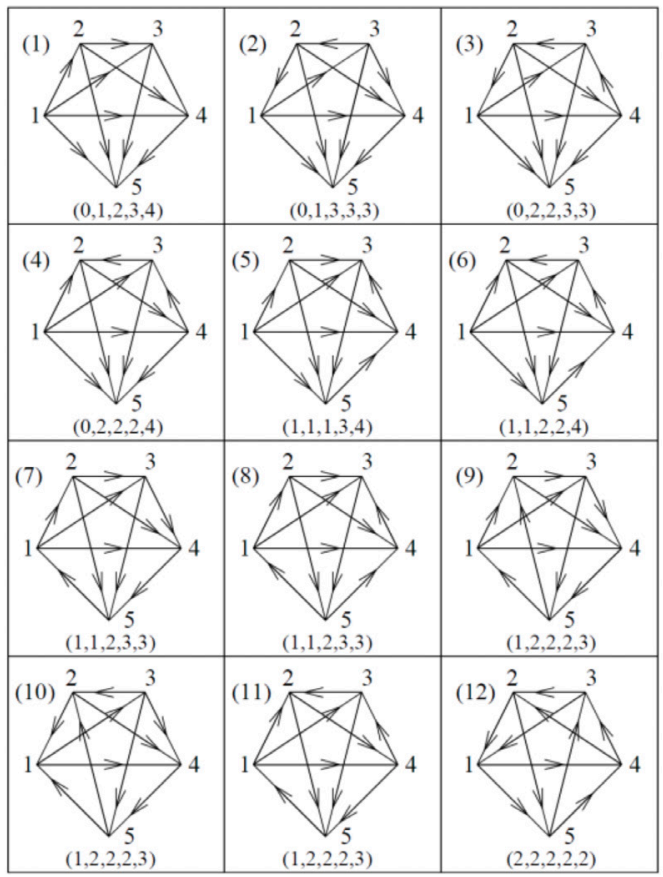

Figura 2. Clases de isomorfismo de 5-torneos. 
Definición 2.3. Dado un torneo $T$, la matriz de adyacencia $M(T)=\left(a_{i j}\right)$ del torneo se define por: $a_{i j}=0$, si $j \rightarrow i ; a_{i j}=1$, si $i \rightarrow j$; y $a_{i i}=0$.

Las matrices de adyacencia de los torneos (2) y (3) en la Figura 1 son respectivamente

$\left(\begin{array}{lll}0 & 1 & 1 \\ 0 & 0 & 1 \\ 0 & 0 & 0\end{array}\right)$ y $\left(\begin{array}{lll}0 & 1 & 0 \\ 0 & 0 & 1 \\ 1 & 0 & 0\end{array}\right)$

Definición 2.4. Un torneo se dice que es parabólico si, salvo permutaciones, es tal que para cada par de vértices $i$ y $j$ se tiene que

$\left\{\begin{array}{l}i \rightarrow j, \quad \text { si } j-i \text { es impar } \\ j \rightarrow i, \quad \text { si } j-i \text { es par }\end{array}\right.$

Estos torneos reciben ese nombre porque están relacionados con las estructuras cuasicomplejas parabólicas sobre variedades bandera (ver [29]). En [29], se probó el siguiente resultado

Teorema 2.1. Si $T$ es un n-torneo parabólico entonces su vector resultado tiene la forma

$(n-k, \ldots, n-k), \quad$ si $n=2 k-1$

$(n-(k+1), \ldots, n-(k+1), n-k, \ldots, n-k), \quad$ si $n=2 k$

La Figura 3 contiene los torneos parabólicos para los casos con 3,4,5 y 6 vértices, respectivamente. La recíproca del teorema anterior es falsa porque, por ejemplo, para $n=6$, existen torneos distintos del parabólico que tienen el mismo vector resultado.

Para el caso en que $n=2 k-1$ o sea impar, el teorema si es una equivalencia pues solo hay una clase de isomorfismo de torneos cuyo vector resultado tiene iguales todas las entradas $(n-k, \ldots, n-k)$. Esto se debe a que la suma de las entradas en el vector resultado debe ser

$1+2+\cdots+n-1=\frac{(n-1) n}{2}=\frac{(2 k-2) n}{2}=(k-1) n=(n-k) n$.

Es decir, tenemos el siguiente resultado

Proposición 2.1. Para $n=2 k-1, T$ es un $n$-torneo parabólico si y solo si su vector resultado tiene la forma $(n-k, \ldots, n-k)$. 
Los torneos objeto de esta proposición son llamados "torneos carrusel" y han sido estudiados por Coregliano, ver [7] y [8].

Definición 2.5. Consideremos un $n$-torneo $T$, dados $x$ y $y$ dos vértices del torneo un camino dirigido de $x$ a $y$ es una secuencia de $\operatorname{arcos}\left\{a_{i} \rightarrow a_{i+1}\right\}_{i=1}^{k}$, donde $a_{1}=x$ y $a_{k}=y$. Decimos que la longitud del camino es $k$.

Se dice que $T$ es fuertemente conexo si para cada par de vértices x,y existe un camino dirigido de $x$ a $y$.

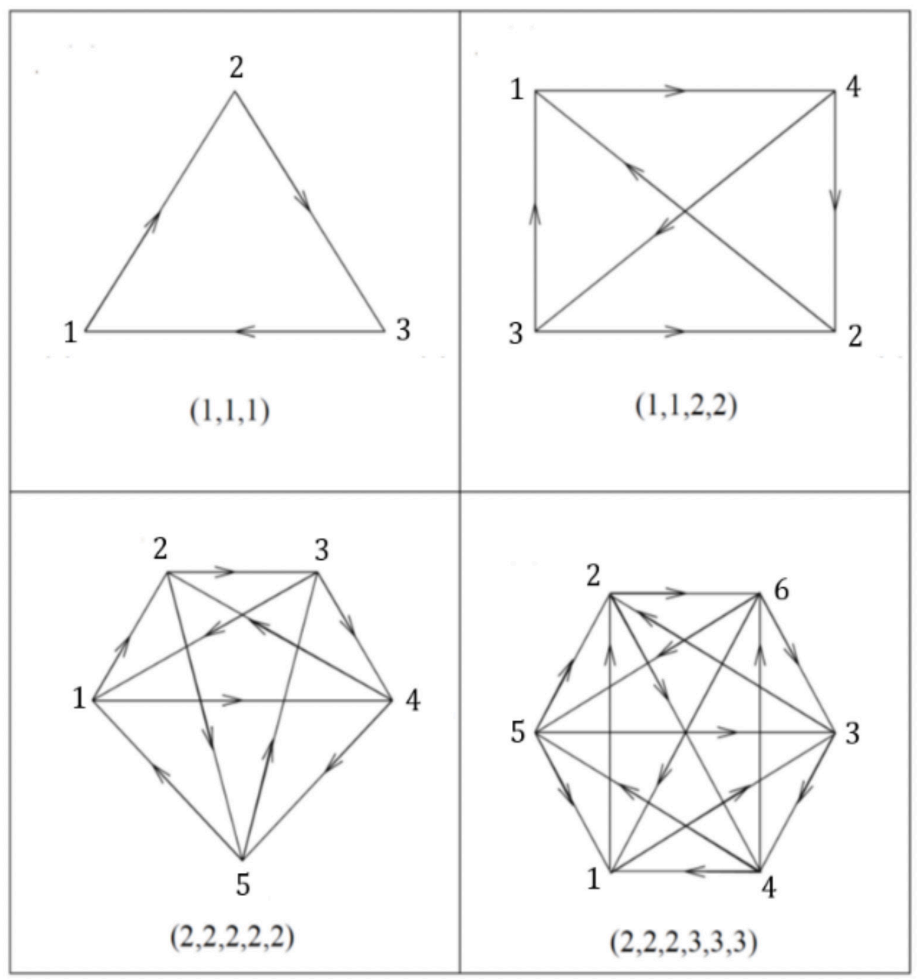

Figura 3. $n$-torneos parabólicos para $n=3,4,5,6$

Definición 2.6. La longitud $d(x, y)$, entre dos vértices $x, y$ del torneo está dada por la longitud del camino dirigido más corto de $x$ a $y$.

Claramente, si $T$ es fuertemente conexo entonces la distancia es finita. La función distancia es positiva y satisface la desigualdad triangular pero no necesariamente es simétrica, es decir, dados dos vértices $x, y$ no necesariamente $\mathrm{d}(\mathrm{x}, \mathrm{y})=\mathrm{d}(\mathrm{y}, \mathrm{x})$. Por ejemplo, en el torneo de la Figura 4, tenemos que $d(2,1)=1 \neq 2=d(1,2)$. 


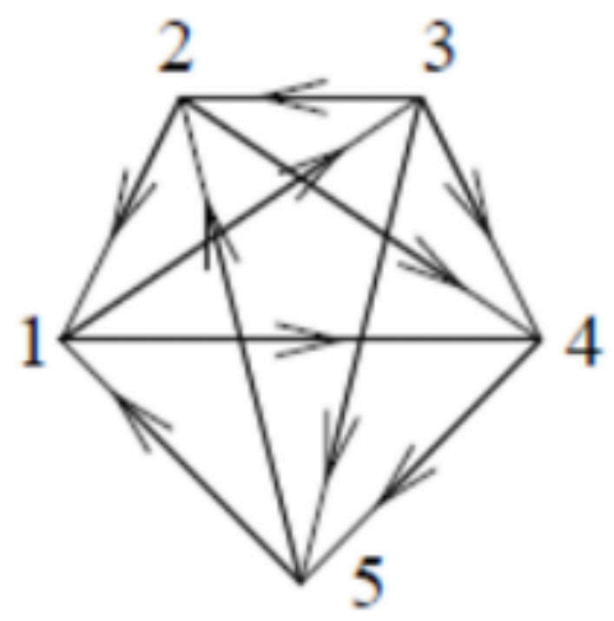

Figura 4. Ejemplo de 5-torneo

Definición 2.7. Para cualquier vértice $x$ de un torneo $T=(V, E)$ definimos la vecindad saliendo de $x$ como el subtorneo

$\Gamma^{\text {out }}(\mathrm{x})=\{y \in V:(x, y) \in E\}$

De forma similar se define la vecindad entrando de $x$ como el subtorneo

$\Gamma^{\text {in }}(\mathrm{x})=\{z \in V:(z, X) \in E\}$

Para cualquier vértice $x \in V$, el grado de salida de $x$, denotado por $d_{x}^{\text {out }}$, es el número de arcos que salen de $x$, esto es, $d_{x}^{\text {out }}=\left|\Gamma^{\text {out }}(x)\right|$. Decimos que $T$ es $d$-regular si cada vértice tiene el mismo grado de salida. De acuerdo con el Teorema 2.1. los $n$-torneos parabólicos con $n$ impar son $d$-regulares.

Similarmente, el grado de entrada de $x$, denotado por $d_{x}^{\text {in }}$, es el número de arcos que llegan (entran) a $x$, esto es $d_{x}^{\text {in }}=\left|\Gamma^{\text {in }}(x)\right|$.

Un tipo de torneos que han sido estudiados y usados para estudiar la geometría de variedades bandera son los torneos localmente transitivos. Un torneo $T=(V, E)$ se dice que es localmente transitivo si para cada vértice $x \in V$ los subtorneos $\Gamma^{\text {out }}(x)$ y $\Gamma^{\text {in }}(x)$ son transitivos, ver $[1,6,11,12]$.

Resulta entonces natural preguntarse si un torneo parabólico es localmente transitivo, esta pregunta no ha sido abordada en otros artículos sobre torneos escritos por el autor de este trabajo por otros autores. 
Teorema 2.2. Si $T$ es un $n$-torneo parabólico con vértices $\{1, \ldots, n\}$ entonces $T$ también es localmente transitivo.

Demostración: Por el teorema 2.1 sabemos que si $T$ es parabólico entonces su vector marcador es

$(n-k, \ldots, n-k), \quad$ si $n=2 k-1$

$\mathrm{O}$

$(n-(k+1), \ldots, n-(k+1), n-k, \ldots, n-k), \quad$ si $n=2 k$.

Supongamos que $n=2 k-1$ entonces T tiene vector marcador igual a $(n-k, \ldots, n-k)$. Por la definición de torneo parabólico tenemos que

$$
\begin{gathered}
n-(n-2)=2 \text { es par } \quad \Rightarrow \quad n \rightarrow n-2 \\
n-(n-4)=4 \text { es par } \quad \Rightarrow \quad n \rightarrow n-4 \\
\vdots \\
\vdots \\
n-3=2 k-1-3=2 k-4 \text { es par } \quad \Rightarrow \quad n \rightarrow 3 \\
n-1=2 k-1-1=2 k-2 \text { es par } \quad \Rightarrow \quad n \rightarrow 1
\end{gathered}
$$

Por tanto,

$\Gamma^{\text {out }}(n)=\{n-2, n-4, \ldots, 3,1\}$

Similarmente podemos ver que

$\Gamma^{i n}(n)=\{n-1, n-3, \ldots, 4,2\}$.

Veamos ahora que $\Gamma^{\text {out }}(n)$ es transitivo:

$$
\begin{array}{ccc}
(n-2)-(n-4)=2 \text { es par } & \Rightarrow & n-2 \rightarrow n-4 \\
(n-2)-(n-6)=4 \text { es par } & \Rightarrow & n-2 \rightarrow n-6 \\
\vdots & & \\
(n-2)-3=2 k-6 \text { es par } & \Rightarrow & n-2 \rightarrow 3 \\
(n-2)-1=2 k-4 \text { es par } & \Rightarrow & n-2 \rightarrow 1
\end{array}
$$


Asimismo se puede ver que $n-4$ le gana a $n-6, n-8 . ., 3,1$ y así sucesivamente con todos los otros vértices hasta que lleguemos a 1 el cual pierde con todos los otros. Es decir que el vector marcador de $\Gamma^{\text {out }}(n)$ es

$$
\left(0,1,2, \ldots, \frac{n-1}{2}\right)
$$

en otras palabras $\Gamma^{\text {out }}(n)$ es transitivo. De forma similar se puede ver que $\Gamma^{\text {in }}(n)$ también es transitivo.

Claramente, lo que hemos hecho hasta aquí es válido para cualquier vértice pues siempre es posible renombrar los vértices de tal forma que el vértice para el cual vamos a calcular sus vecindades entrando o saliendo sea el vértice $n$. Finalmente, un argumento similar funciona si $n=2 k$. Por tanto, cualquier torneo parabólico es localmente transitivo.

\section{La curvatura de Ricci}

Esta sección se escribió siguiendo algunas ideas contenidas en el interesante artículo de Yamada [31], publicado recientemente.

Definición 3.1. Dado un torneo $T=(V, E)$, definimos para cualquier vértice $x \in V$ y cualquier $\alpha \in[0,1]$ una medida de probabilidad $m_{x}^{\alpha}$ sobre $V$ como

$$
m_{x}^{\alpha}(v)=\left\{\begin{aligned}
\alpha, & \text { si } v=x \\
\frac{1-\alpha}{d_{x}^{\text {out }},} & \text { si }(x, v) \in E \\
0, & \text { en otro caso }
\end{aligned}\right.
$$

Definición 3.2. Dadas dos medidas de probabilidad $\mu$ y $v$ sobre $V$ definimos la distancia de Wasserstein entre $\mu$ y $v$ como

$$
W(\mu, v)=\inf _{A} \sum_{u, v} A(u, v) d(u, v)
$$

donde $\mathrm{A}: \mathrm{V} \times \mathrm{V} \rightarrow[0,1]$ son todas las aplicaciones tales que

$$
\left\{\begin{array}{l}
\sum_{v \in V} A(u, v)=\mu(u) \\
\sum_{u \in V} A(u, v)=v(v)
\end{array}\right.
$$

Una aplicación como esta es llamada un acoplamiento entre $\mu$ y v. Para tomar el ínfimo en la definición anterior es necesario garantizar la existencia de un acoplamiento, Yamada [31] define el siguiente 
acoplamiento entre dos medidas de probabilidad $m_{x}^{\alpha}$ y $m_{y}^{\alpha}$ la cual satisface (10)

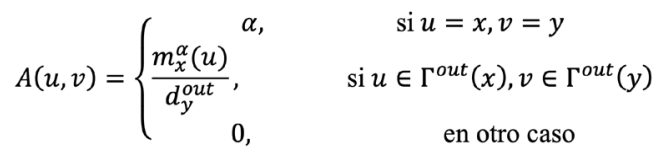

Como la distancia no es simétrica un acoplamiento A para el cual se alcanza la distancia de Wasserstein no necesariamente existe. En caso de existir es llamado el acoplamiento óptimo.

Definición 3.3. Dado un torneo $T=(V, E)$, para cualesquiera dos vértices $\mathrm{x}, \mathrm{y} \in \mathrm{V}$ se define la $\alpha$-curvatura de Ricci de $x$ y $y$ como

$\kappa_{\alpha}(x, y)=1-\frac{W\left(m_{x}^{\alpha}, m_{y}^{\alpha}\right)}{d(x, y)}$

En el artículo de Yamada [31], usando unas técnicas un tanto sofisticadas, se prueba la existencia del siguiente límite

$\lim _{\alpha \rightarrow 1} \frac{\kappa_{\alpha}(x, y)}{1-\alpha}$

para cualquier par de vértices $x, y$. La existencia de dicho límite justifica la siguiente

Definición 3.4. Dado un torneo $T=(V, E)$, para cualesquiera dos vértices $x, y \in V$ se define la curvatura de Ricci de $x$ y $y$ como

$\kappa(x, y)=\lim _{\alpha \rightarrow 1} \frac{\kappa_{\alpha}(x, y)}{1-\alpha}$

\section{Curvatura de Ricci para torneos parabólicos}

En esta sección encontramos la curvatura de Ricci de los torneos parabólicos d-regulares, es decir, torneos parabólicos para los que cada vértice tiene el mismo grado de salida. Estos torneos son aquellos torneos $T$ con $n=2 k-1$ vértices cuyo vector marcador es $(n-k, \ldots, n-k)$. Estas son las matrices de adyacencia de los correspondientes torneos para $n=3,5,7,9$ 
$n=3: \quad\left(\begin{array}{lll}0 & 1 & 0 \\ 0 & 0 & 1 \\ 1 & 0 & 0\end{array}\right), \quad$ vector marcador $=(1,1,1)$

$n=5: \quad\left(\begin{array}{lllll}0 & 1 & 1 & 0 & 0 \\ 0 & 0 & 1 & 1 & 0 \\ 0 & 0 & 0 & 1 & 1 \\ 1 & 0 & 0 & 0 & 1 \\ 1 & 1 & 0 & 0 & 0\end{array}\right), \quad$ vector marcador $=(2,2,2,2,2)$

$n=7:\left(\begin{array}{lllllll}0 & 1 & 1 & 1 & 0 & 0 & 0 \\ 0 & 0 & 1 & 1 & 1 & 0 & 0 \\ 0 & 0 & 0 & 1 & 1 & 1 & 0 \\ 0 & 0 & 0 & 0 & 1 & 1 & 1 \\ 1 & 0 & 0 & 0 & 0 & 1 & 1 \\ 1 & 1 & 0 & 0 & 0 & 0 & 1 \\ 1 & 1 & 1 & 0 & 0 & 0 & 0\end{array}\right), \quad$ vector marcador $=(3,3,3,3,3,3,3)$

$n=9:\left(\begin{array}{lllllllll}0 & 1 & 1 & 1 & 1 & 0 & 0 & 0 & 0 \\ 0 & 0 & 1 & 1 & 1 & 1 & 0 & 0 & 0 \\ 0 & 0 & 0 & 1 & 1 & 1 & 1 & 0 & 0 \\ 0 & 0 & 0 & 0 & 1 & 1 & 1 & 1 & 0 \\ 0 & 0 & 0 & 0 & 0 & 1 & 1 & 1 & 1 \\ 1 & 0 & 0 & 0 & 0 & 0 & 1 & 1 & 1 \\ 1 & 1 & 0 & 0 & 0 & 0 & 0 & 1 & 1 \\ 1 & 1 & 1 & 0 & 0 & 0 & 0 & 0 & 1 \\ 1 & 1 & 1 & 1 & 0 & 0 & 0 & 0 & 0\end{array}\right)$, vector marcador $=(4,4,4,4,4,4,4,4,4)$

Tomemos el último caso para hacer algunos cálculos que nos permitan encontrar la curvatura de Ricci, llamemos $v_{1^{\prime}}, v_{2}, v_{3}, v_{4}, v_{5}, v_{6}, v_{7}, v_{8}, v_{9}$ los vértices del torneo entonces las vecindades saliendo para cada vértice son

$$
\begin{aligned}
& \Gamma^{\text {out }}\left(v_{1}\right)=\left\{v_{2}, v_{3}, v_{4}, v_{5}\right\} \\
& \Gamma^{\text {out }}\left(v_{2}\right)=\left\{v_{3}, v_{4}, v_{5}, v_{6}\right\} \\
& \Gamma^{\text {out }}\left(v_{3}\right)=\left\{v_{4}, v_{5}, v_{6}, v_{7}\right\} \\
& \Gamma^{\text {out }}\left(v_{4}\right)=\left\{v_{5}, v_{6}, v_{7}, v_{8}\right\} \\
& \Gamma^{\text {out }}\left(v_{5}\right)=\left\{v_{6}, v_{7}, v_{8}, v_{9}\right\} \\
& \Gamma^{\text {out }}\left(v_{6}\right)=\left\{v_{1}, v_{7}, v_{8}, v_{9}\right\} \\
& \Gamma^{\text {out }}\left(v_{7}\right)=\left\{v_{1}, v_{2}, v_{8}, v_{9}\right\} \\
& \Gamma^{\text {out }}\left(v_{8}\right)=\left\{v_{1}, v_{2}, v_{3}, v_{9}\right\} \\
& \Gamma^{\text {out }}\left(v_{9}\right)=\left\{v_{1}, v_{2}, v_{3}, v_{4}\right\}
\end{aligned}
$$

Claramente, vemos que para $\mathrm{j} \in\{2, \ldots, \mathrm{k}-1\}$ tenemos

$\Gamma^{\text {out }}\left(v_{1}\right) \cap \Gamma^{\text {out }}\left(v_{j}\right)=\left\{v_{j+1}, \ldots, v_{k}\right\} \mathrm{y} \Gamma^{\text {out }}\left(v_{1}\right) \cap \Gamma^{\text {out }}\left(v_{k+1}\right)=\emptyset$

Calculemos la curvatura sobre los arcos saliendo de v1, entonces para $j \in\{2, \ldots, k\}$ y $l \in\{1, \ldots, j-1\}$, se define un acoplamiento $A_{j}$ entre $m_{v_{1}}^{\alpha}$ y $m_{v_{j}}^{\alpha}$ como 
$A_{j}(u, v)=\left\{\begin{array}{rc}\alpha, & \text { si } u=v_{1}, v=v_{j} \\ \frac{1-\alpha}{n}, & \text { si } u=v \in \Gamma^{o u t}\left(v_{1}\right) \cap \Gamma^{o u t}\left(v_{j}\right) \\ \frac{1-\alpha}{n}, & \text { si } u=v_{1+l}, v_{k+l} \\ 0, & \text { en otro caso }\end{array}\right.$

y la función $f_{j:} \Gamma^{\text {out }}\left(v_{1}\right) \rightarrow \Gamma^{\text {out }}\left(v_{j}\right)$ dada por

$f_{j}(u)=\left\{\begin{array}{rr}1, & \text { si } u=v_{1} \\ -1, & \text { si } u \in \Gamma^{o u t}\left(v_{j}\right) \backslash \Gamma^{o u t}\left(v_{1}\right) \\ 0, & \text { en otro caso }\end{array}\right.$

Observamos que usando el acoplamiento $A_{j}$ y la función $f j$, la curvatura para cualquier arco $(x, y) \in E$ la curvatura es alguno de los siguientes valores

$0, \frac{1}{k-1}, \ldots, \frac{k-2}{k-1}$.

Teorema 4.1. Dado un torneo $T=(V, E)$ parabólico d-regular con $n=2 k-1$ vértices, la curvatura $\kappa(x, y)$ para cualquier arco $(x, y) \in E$ es alguno de los siguientes valores

$0, \frac{1}{k-1}, \ldots, \frac{k-2}{k-1}$

\section{Conclusiones}

Este trabajo nos presenta una nueva posibilidad para adelantar nuevos trabajos de investigación usando el concepto de curvatura de Ricci para grafos introducido por Lin, Lu y Yau[14]. El objetivo seríatratar deconectar el nuevo concepto con nuevas propiedades geométricas de las variedades bandera. En particular deberíamos estudiar más a fondo la definición de curvatura de Ricci para grafos dirigidos introducida por Yamada en [31] a partir del trabajo de Lin, Lu y Yau[14].

Una idea para continuar este trabajo es seguir explorando este concepto para el caso de torneos transitivos y localmente transitivos e intentar obtener nuevos resultados para estructuras cuasicomplejas y por tanto para métricas (1,2)-simplécticas sobre las variedades bandera. A partir de esto posteriormente, se debería intentar generalizar estos resultados para $f$-estructuras (ver [3]) sobre las variedades bandera y por tanto buscar nuevas propiedades para métricas sobre las variedades bandera definidas usando $f$-estructuras.

Por otro lado, también sería interesante continuar estudiando los grafos como tal y las diferentes curvaturas que se pueden definir para grafos. Por ejemplo, la curvatura de Forman-Ricci introducida por Leal et al. en [13], la cual se basa en el trabajo de Forman [10], parece estar relacionado con un concepto bastante conocido en geometría llamado flujo de Ricci. 
Más recientemente, Monsalve et al. $[18,19]$ han obtenido resultados muy interesantes sobre energía de grafos dirigidos en los cuales se usa fuertemente el grado de salida $d_{x}^{\text {out }}$ y el grado de entrada $d_{x}^{\text {in }}$ de los vértices del grafo. La idea es estudiar estos artículos y tratar de relacionar los resultados allí presentados con el concepto de curvatura de Ricci o de Forman-Ricci.

\section{Referencias}

[1] A. E. Brouwer, "The enumeration of locally transitive tournaments", AfdelingZuivereWiskunde [Department of Pure Mathematics], 138, Mathematisch Centrum, Amsterdam, 1980.

[2] F.E. Burstall\& S. Salamon, “Tournaments, Flags and Harmonic Maps”, Math. Ann. 277, 1987, $249-265$.

[3] N. Cohen, C. Negreiros, M. Paredes, S. Pinzón and L. San Martin, "f-Structures on the classical flag manifold which admit $(1,2)$-symplectic metrics”, Tohoku Mathematical Journal Vol. 57, No. 2 (2005), 261-271.

[4] N. Cohen, C. J. C. Negreiros\& L. A. B. San Martin, “(1, 2)-Symplectic metrics, flag manifolds and tournaments", Bull. London Math. Soc. 34, 2002, 1-9.

[5] N.Cohen, C.J.C.Negreiros\&L.A.B.San Martin,“A rank-three condition for invariant (1,2)-symplectic almost Hermitian structures on flag manifolds”, Bull. Braz. Math. Soc. Nerw Series 33(1), 2002, 49-73.

[6] N. Cohen, M. Paredes \& S. Pinzón, "Locally transitive tournaments and (1,2)-symplectic metrics on maximal flag manifolds", Illinois Journal of Mathematics, 48(4), 2004, 1405-1415.

[7] L.N. Coregliano, "Quasi-carousel tournaments", Journal of Graph Theory, 88(1), 2018, 192-210.

[8] L. N. Coregliano, "Flag algebras and tournaments",Dissertaçãode Mestrado, Universidade de São Paulo, 2015.

[9] E. Flórez, "Introducción a la teoría de torneos", Trabajo de grado, Licenciatura en Matemáticas, Universidad Industrial de Santander, Bucaramanga, Colombia, 2001.

[10] R. Forman, "Bochner's method for cell complexes and combinatorial Ricci curvature", Discrete and Computational Geometry, 29(3), 2003, 323-374.

[11] J.S. Fuentes, M. Paredes and S. Pinzón, "Acerca de los dígrafos localmente transitivos", Revista Integración Vol. 22 (2004), 23-35. 
[12] J.S. Fuentes, "Digrafos localmente transitivos”, Trabajo de grado, Licenciatura en Matemáticas, Universidad Industrial de Santander, Bucaramanga, Colombia, 2004.

[13] W. Leal, G. Restrepo, P.F. Stadler\& J. Jost, "Forman-Ricci Curvature for Hypergraphs", 2018. arXiv preprint arXiv:1811.07825.

[14] Y. Lin, L. Lu \& S.T. Yau, "Ricci curvature of graphs", Tohoku Mathematical Journal, Second Series, 63(4), 2011, 605-627.

[15] Y. Lin, L. Lu \& S.T. Yau, "Ricci-flat graphs with girth at least five", 2013, arXiv preprint arXiv:1301.0102.

[16] Y. Lin \& S.T. Yau, "Ricci Curvature and eigenvalue estimate on locally finite graphs", Math. Res. Lett. 17, 2010, 343-356.

[17] X. Mo \& C.J.C. Negreiros, “(1,2)-symplectic structures on flag manifolds”,Tohoku Mathematical Journal, Second Series, 52(2), 2000, 271-282.

[18] J. Monsalve, J. Rada, "Oriented bipartite graphs with minimal trace norm”, Linear Multilinear Algebra 67 (6) (2019) 1121-1131.

[19] J. Monsalve, J. Rada \& Y. Shi, "Extremal values of energy over oriented bicyclic graphs”, Applied Mathematics and Computation 342 (2019) 26-34.

[20] J. W. Moon, “Topics on tournaments”, Holt, Rinehart and Winston, New York, 1968.

[21] Y. Ollivier, "Ricci curvature of Markov chains on metric spaces", Journal of Functional Analysis, 256, 2009, 810-864.

[22] M. Paredes, "Aspectos da Geometria Complexa das Variedades Bandeira”, Tese de Doutorado, Universidade Estadual de Campinas, Brasil, 2000.

[23] M. Paredes, "Some results on the geometry of full flag manifolds and harmonic maps", RevistaColombianade Matemáticas 34, 2000, 57-89.

[24] M. Paredes, “Torneos y estructuras parabolicas sobre variedades bandera maximales”, Revista Integración Vol. 17, No. 1, 1999, 1-10. 
[25] M. Paredes, "Families of (1,2)-symplectic metrics on full flag manifolds", Int.J. Math. Math. Sci. $29,2002,651-664$.

[26] M. Paredes, P. González \& B. McKay, "Sobre un tipo especial de torneos y una clase de métricas sobre variedades bandera”, Memorias de la Primera Conferencia Iberoamericana de Matemática Computacional, Sociedad Colombiana de Matemáticas, Bogotá, 2001.

[27] M. Paredes \& S. Pinzón,"On the codifferential of the Kähler form and cosymplectic metrics on maximal flag manifolds”, Turkish Journal of Mathematics 34, 2010, 305-316.

[28] M. Paredes\& S. Pinzón, "Some remarks about cosymplectic metrics on maximal flag manifolds", in Geometric and Topological Methods in Quantum Field Theory, 394-404, Cambridge University Press, 2010.

[29] M. Paredes\& S. Pinzón, "Tournaments and parabolic almost complex structures on flag manifolds", Contemporary Mathematics, Vol. 509, 2010, 221-231.

[30] M. Paredes \& S. Pinzón, "Variedades bandera maximales, torneos y aplicaciones armónicas", Revista Integración, Vol 18, No. 2, 2000, 65-77.

[31] T. Yamada, "The Ricci curvature on directed graphs", Journal of the Korean Mathematical Society, 56(1), 2019, 113-125. 